\title{
Cardiac 7-transmembrane-spanning domain receptor portfolios: diversify, diversify, diversify
}

\author{
Stephen B. Liggett
}

Cardiopulmonary Genomics Program, University of Maryland School of Medicine, Baltimore, Maryland, USA.

\begin{abstract}
Enhanced signaling in myocytes by the $G$ protein $G_{q}$ has been implicated in cardiac hypertrophy and the transition to heart failure. $\alpha_{1}$-Adrenergic receptors $\left(\alpha_{1}\right.$-ARs) are members of the 7-transmembrane-spanning domain (7-TM) receptor family and signal via interaction with $G_{q}$ in the heart. The specific effects of a loss of $\alpha_{1}$-AR signaling in the heart are explored by O'Connell et al. in this issue of the JCI (see the related article beginning on page 1005). Paradoxically, gene ablation of the $\alpha_{1 \mathrm{~A}}$ and $\alpha_{1 \mathrm{~B}}$ subtypes in mice results in a maladaptive form of reactive cardiac hypertrophy from pressure overload, with a predisposition to heart failure. Thus signaling to the $\alpha_{1}$-AR (compared with signaling from other receptors such as angiotensin receptors, which also couple to $G_{q}$ ) appears to be specifically required for a normal hypertrophic response. This represents another example of how receptors that share common $G$ proteins have diversified, developing unique signaling programs. These findings may have particular clinical relevance because of the widespread use of $\alpha_{1}$-AR antagonists in the treatment of hypertension and symptomatic prostate enlargement.
\end{abstract}

It is now recognized that there are 2 distinct subgroups of $\alpha$-adrenergic receptors, designated as $\alpha_{1}$-adrenergic receptors ( $\alpha_{1}$-ARs) and $\alpha_{2}$-ARs, all of which are members of the superfamily of 7-transmembrane-spanning domain (7-TM) receptors (also termed $\mathrm{G}$ protein-coupled receptors). There are 3 human $\alpha_{1}$-AR subtypes, denoted $\alpha_{1 \mathrm{~A}}, \alpha_{1 \mathrm{~B}}$, and $\alpha_{1 D}$. Since $\alpha_{1}$-ARs expressed on vascular smooth muscle act to constrict and thus increase peripheral vascular resistance, there has been substantial development and widespread use of $\alpha_{1}$-AR antagonists for the treatment of hypertension. What has not been well acknowledged is the fact that $\alpha_{1}$-ARs are also expressed on cardiomyocytes, and thus treatment of hypertension with $\alpha_{1}$-AR antagonists may also have effects on the heart that are distinct from afterload reduction. All $\alpha_{1}$-AR subtypes couple to the heterotrimeric $G$ protein $\mathrm{G}_{\mathrm{q}}$. Upon agonist activation, the $\mathrm{G}_{\alpha}$ subunit activates the effector phospholipase C, which produces at least 2 intracellular second messengers, inositol-1,4,5-tri-

Nonstandard abbreviations used: AR, adrenergic receptor; $\mathrm{AT}_{1}$, angiotensin II type 1; TAC, transverse aortic constriction; 7-TM, 7-transmembrane-spanning domain.

Conflict of interest: The author has declared that no conflict of interest exists.

Citation for this article: J. Clin. Invest. 116:875-877 (2006). doi:10.1172/JCI28234. phosphate and diacylglycerol. The former increases intracellular calcium, while the latter activates several PKC isoenzymes that modify heart failure (1). Since catecholamines are elevated in heart failure, cardiac $\alpha_{1}-A R / G_{q}$ signaling is activated to various extents in the syndrome.

The $G_{\alpha q}$ pathway has been studied extensively as to its role in cardiac hypertrophy and heart failure (2). Cardiac overexpression of $G_{\alpha q}$ in transgenic mice (3) results in hypertrophy, decreased ventricular function, loss of $\beta$-adrenergic receptor inotropic responsiveness, and induction of a classic hypertrophy gene expression profile. In these mice, pressure overload by surgical transverse aortic constriction (TAC), pregnancy, or higher transgenic overexpression of $\mathrm{G}_{\alpha \mathrm{q}}$ resulted in cardiomyocyte apoptosis and decompensated heart failure (3, 4). Other studies showed that transgenic overexpression of a $\mathrm{G}_{\alpha \mathrm{q}}$ dominant-negative minigene resulted in the lack of a hypertrophy response to TAC (5). Furthermore, cardiac overexpression of a constitutively activated $\alpha_{1 \mathrm{~B}}$-AR resulted in cardiac hypertrophy (6), while a more severe cardiomyopathy developed as a result of overexpression of the $\mathrm{G}_{\mathrm{q}}$-coupled angiotensin II type $1\left(\mathrm{AT}_{1}\right)$ receptor $(7)$. These studies, then, began to point toward hyperactive $G_{\alpha q}$ signaling as a key mechanism causing hypertrophy, depressed ventricular function, and failure.
A readily drawn conclusion from such studies might be that in the human heart, factors that increase $G_{\alpha q}$ signaling predispose to cardiac hypertrophy and, potentially, the transition from hypertrophy to decompensated heart failure. In addition, approaches that decrease this signaling might be protective against the development of heart failure or be beneficial in treatment.

\section{Ablation of $\alpha_{1}$-ARs and cardiac hypertrophy}

In the report by O'Connell et al. in this issue of the JCI (8), the hypertrophic response to TAC was assessed in mice in which the genes encoding $\alpha_{1 \mathrm{~A}}$-AR and $\alpha_{1 \mathrm{~B}}$-AR had been ablated ( $\alpha_{1 \mathrm{~A} / \mathrm{B}} \mathrm{KO}$ mice). Mice without these $G_{\alpha q}$-coupled receptors demonstrated rapid decompensation and heart failure after TAC. In those that survived, echocardiographic studies showed lower ejection fractions than in WT mice. Although both sets of mice exhibited hypertrophy, the $\alpha_{1 \mathrm{~A} / \mathrm{B}} \mathrm{KO}$ mice had increased apoptosis and interstitial fibrosis. Furthermore, they had an atypical hypertrophy-associated gene profile, with minimal changes in expression of $\beta$-myosin heavy chain, $\alpha$-skeletal actin, and atrial natriuretic factor transcripts. These data suggest that $\alpha_{1}-A R / G_{q}$ signaling is necessary for adaptation to pressure overload. This issue is of substantial clinical importance because of the extensive use of $\alpha_{1}$-AR antagonists for the treatment of hypertension and symptomatic prostate enlargement. In a large cohort of hypertensive patients, those treated with the $\alpha_{1}$-AR antagonist doxazosin had a relative risk of $2.04(95 \%$ confidence interval $=1.79-2.32$, $P<0.001)$ of developing heart failure compared with those receiving a diuretic (9). Other studies with smaller cohorts have also observed this relationship but indicate an attenuation of this risk after adjustment for systolic blood pressure (10). Of note, this latter study found that systolic blood pressure was lower in the diuretic group compared with the $\alpha_{1}$-AR antagonist group, particularly in women, in whom the risk of heart failure was greatest. This may 

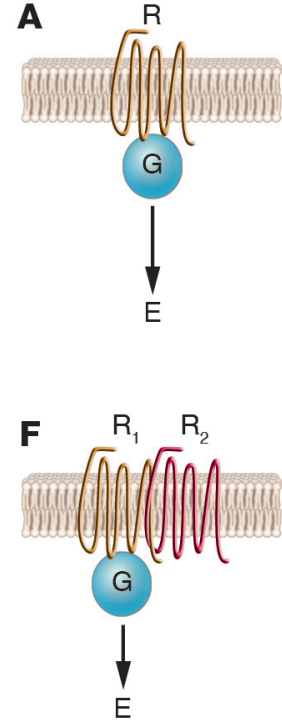
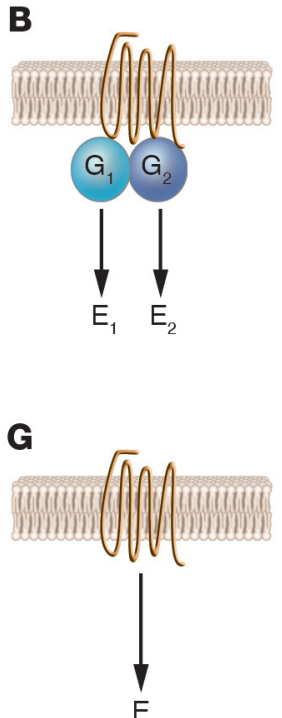
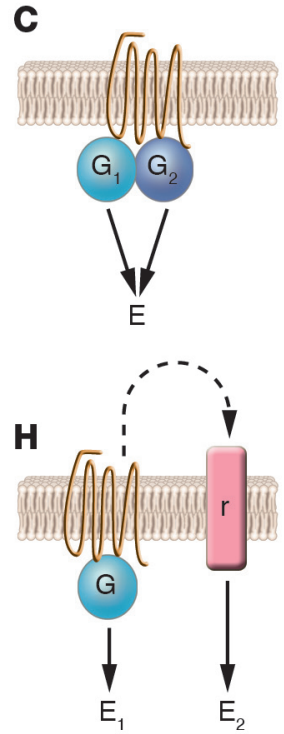
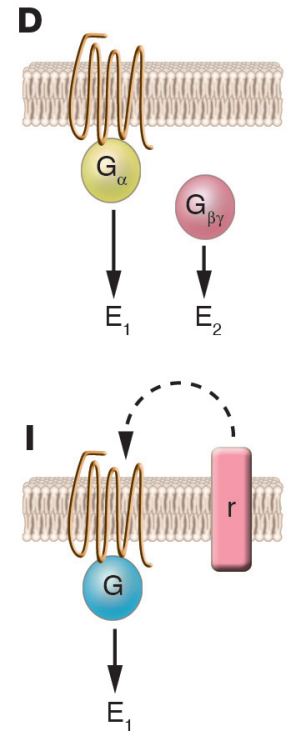
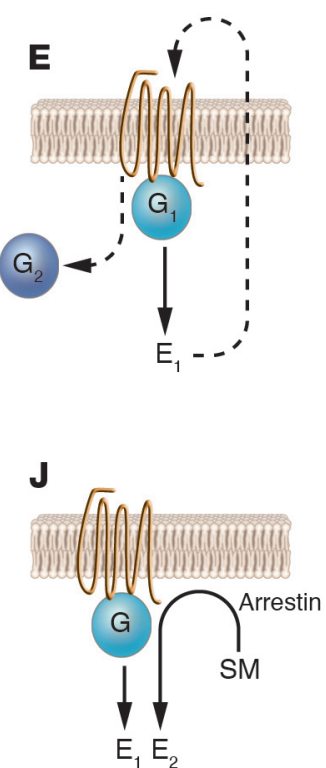

Figure 1

Mechanisms of 7-TM receptor diversification. The classic paradigm is shown in A, where a receptor (denoted "R," "R1," or "R2") couples to a G protein (denoted "G"), which activates an effector (denoted "E"). In B, 1 receptor couples to 2 G proteins, thereby activating 2 effectors. In C, receptor coupling to $2 \mathrm{G}$ proteins modulates a single effector. Scenario $\mathbf{D}$ involves coupling to a single $\mathrm{G}$ protein, but the $\alpha$ and $\beta \gamma$ subunits carry out distinct signaling to 2 different effectors. In E, feedback modulation of the receptor (such as phosphorylation) by 1 effector (or an effector-activated event such as protein kinase activation) causes the receptor to couple to the second $\mathrm{G}$ protein. In $\mathbf{F}$, the functional coupling of $\mathrm{R}_{1}$ to the $\mathrm{G}$ protein is altered by its heterodimerization with another receptor, $\mathrm{R}_{2}$. In contrast to the above, 7-TM receptors can couple directly to effectors without the $\mathbf{G}$ protein intermediary $(\mathbf{G})$. In $\mathbf{H}$ and $\mathbf{I}$, the 7-TM receptor is affected by, or acts upon, other types of receptors (denoted " $r$ "), such as receptor tyrosine kinases, which alter signaling via the respective cognate pathways. In J, a signaling moiety (SM) is redistributed to a microdomain by the scaffolding action of the arrestins. These proteins act both to partially desensitize the receptor and to evoke a second effector signal by this scaffolding. One or more of these mechanisms may be responsible for the specialized signaling of the $\alpha_{1}$-AR required for normal hypertrophy, as elucidated by O'Connell et al. (8).

indicate that normotensive men treated with $\alpha_{1}$-AR antagonists for symptomatic prostatic enlargement are not at significantly increased risk for developing heart failure, but this hypothesis has not been tested in adequately powered trials. Of particular concern would be the scenario in which an individual taking $\alpha_{1}$-AR antagonists develops a stressed myocardium (e.g., new-onset hypertension, myocardial infarction). The inability to develop the appropriate hypertrophic response could predispose such individuals to cardiac failure.

\section{A matter of timing}

The data obtained from this study of $\alpha_{1 \mathrm{~A} / \mathrm{B}}$ KO mice (8) provide additional support for the potential deleterious effects of $\alpha_{1}$-AR blockade. How, though, are these results reconciled with the generally perceived notion that enhanced (as opposed to suppressed) receptor $/ G_{\alpha q}$ signaling evokes hypertrophy and/or failure? There are several components to this issue. First, examination of earlier mouse studies (3) indicates that the effects observed in the $G_{\alpha q}$ mouse appear to be mostly due to $G_{\alpha q}$ overexpres- sion being present from birth (because of the properties of the $\alpha$-myosin light chain promoter that was used in the transgenic construct). When an inducible promoter was used, and $G_{\alpha q}$ overexpression was induced after the heart had reached normal size, no pathologic effect was noted (11). In other studies, mice lacking $G_{\alpha q}$ (and the similarly functioning $G_{\alpha 11}$ protein) died in utero with underdeveloped, hypoplastic hearts (12). So, it appears that for cardiac development (one form of growth), a certain critical level of $G_{\alpha q}$ signaling is necessary to achieve normal morphology. $\mathrm{G}_{\alpha \mathrm{q}} /$ $\mathrm{G}_{\alpha 11} \mathrm{KO}$ mice that survive the perinatal period are unable to mount a hypertrophic response to TAC as adults (13).

Thus, similarly to the requirements for normal growth during development, finely tuned $G_{\alpha q}$ signaling appears to be necessary for reactive growth. Taking these studies into account, then, the O'Connell report (8) does not fully define the role of $\alpha_{1}$-AR signaling in the hypertrophic or failure response to pressure overload, because the $\alpha_{1 \mathrm{~A} / \mathrm{B}} \mathrm{KO}$ mice have abnormal hearts as adults. Prior to TAC, these hearts are small- er with decreased myocyte surface area and volume (14). So whether the lack of $\alpha_{1}$-AR signaling in the normal, adult heart has an effect on hypertrophy or the transition to failure cannot be fully addressed in mice that have a lack of $\alpha_{1 \mathrm{~A} / \mathrm{B}}$-AR from conception. An inducible, cell type-specific knockout would be required to explore this issue in the most rigorous manner. Secondly, it is important to realize that cardiac hypertrophy per se is a form of growth whereby the heart responds to states, such as the increased afterload from hypertension, in order to maintain performance. Thus, under these circumstances hypertrophy can be considered as a compensatory and initially advantageous response of the heart. The inability to achieve the normal biochemical and anatomic hypertrophy response, such as when $G_{\alpha q}$ signaling is suppressed, may thus predispose to maladaptive hypertrophy and a more rapid transition to pump failure.

\section{7-TM receptor diversification}

It is not entirely clear why $\alpha_{1}-A R / G_{\alpha q}$ signaling is specifically necessary for a normal 
response to pressure overload. There are numerous other cardiac neurohumoral receptors that also couple to $G_{\alpha q}$ and are activated in heart failure. These include the $\mathrm{AT}_{1}$ receptor, which is an effective pharmacologic target for angiotensin-converting enzyme inhibitors (15) and angiotensin receptor antagonists (16) in the treatment of heart failure. What is apparent, though, is that 7-TM signaling is not a simple, linear, 1-way "switch." Indeed, in the most straightforward model, the hundreds of 7-TM receptors couple to effectors by only a handful of $G$ proteins. So a major question is how receptor subtypes within cells have evolved to carry out specialized functions. Such "signaling diversification" has been shown to be due to a variety of mechanisms (Figure 1). At issue now for the hypertrophy-related 7-TM receptors is to understand how their signaling has differentiated such that specific influences on cardiac function arise. Indeed, the $\alpha_{1 \mathrm{~B}}-\mathrm{AR}$ appears to be in a signaling complex that includes EGFR and PDGFR, which when activated modulate $\alpha_{1 \mathrm{~B}}$-AR function (17). The $\mathrm{G}_{\mathrm{q}}$-coupled $\mathrm{AT}_{1}$ receptor may be in a different complex with the EGFRs, where each receptor can counterregulate the other (18). Thus these receptor complexes must be delineated and their effects as a unit considered, rather than pathophysiologic effects being ascribed to a single member of the unit. The challenge for the future, then, is to think in terms of signaling complexes, rather than individual components, in order to ascertain critical intracellular events in myocytes that modulate the hypertrophic response and transition to failure.

Address correspondence to: Stephen B. Liggett, University of Maryland School of Medicine, 20 Penn Street, HSF-II, Room S-114, Baltimore, Maryland 21201, USA. Phone: (410) 706-6256; Fax: (410) 7066262; E-mail: sligg001@umaryland.edu.

1. Dorn, G.W., and Force, T. 2005. Protein kinase cascades in the regulation of cardiac hypertrophy. J. Clin. Invest. 115:527-537. doi:10.1172/ JCI200524178.

2. Dorn, G.W. 2005. Physiologic growth and pathologic genes in cardiac development and cardiomyopathy. Trends Cardiovasc. Med. 15:185-189.

3. D’Angelo, D.D., et al. 1997. Transgenic Gaq overexpression induces cardiac contractile failure in mice. Proc. Natl. Acad. Sci. U. S. A. 94:8121-8126.

4. Sakata, Y., Hoit, B.D., Liggett, S.B., Walsh, R.A., and Dorn, G.W., II. 1998. Decompensation of pressureoverload hypertrophy in Gaq-overexpressing mice. Circulation. 97:1488-1495.

5. Akhter, S.A., et al. 1998. Targeting the receptor-Gq interface to inhibit in vivo pressure overload myocardial hypertrophy. Science. 280:574-577.

6. Milano, C.A., et al. 1994. Myocardial expression of a constitutively active alpha $1 \mathrm{~B}$-adrenergic receptor in transgenic mice induces cardiac hypertrophy. Proc. Natl. Acad. Sci. U. S. A. 91:10109-10113.

7. Hein, L., et al. 1997. Overexpression of angiotensin AT1 receptor transgene in the mouse myocardium produces a lethal phenotype associated with myocyte hyperplasia and heart block. Proc. Natl. Acad. Sci. U. S. A. 94:6391-6396.

8. O'Connell, T.D., et al. 2006. $\alpha_{1}$-Adrenergic receptors prevent a maladaptive cardiac response to pressure overload. J. Clin. Invest. 116:1005-1015. doi:10.1172/JCI22811.

9. ALLHAT Collaborative Research Group. 2000. Major cardiovascular events in hypertensive patients randomized to doxazosin vs chlorthalidone. JAMA. 283:1967-1975.

10. Bryson, C.L., et al. 2004. Risk of congestive heart failure in an elderly population treated with peripheral alpha-1 antagonists. J. Am. Geriatr. Soc. 52:1648-1654.

11. Syed, F., et al. 2004. Physiological growth synergizes with pathological genes in experimental cardiomyopathy. Circ. Res. 95:1200-1206.

12. Offermanns, S., et al. 1998. Embryonic cardiomyocyte hypoplasia and craniofacial defects in $\mathrm{G}$ alpha q/G alpha 11-mutant mice. EMBOJ. 17:4304-4312.

13. Wettschureck, N., et al. 2001. Absence of pressure overload induced myocardial hypertrophy after conditional inactivation of Galphaq/Galpha11 in cardiomyocytes. Nat. Med. 7:1236-1240.

14. O'Connell, T.D., et al. 2003. The $\alpha(1 \mathrm{~A} / \mathrm{C})$ - and $\alpha(1 \mathrm{~B})$-adrenergic receptors are required for physiological cardiac hypertrophy in the double-knockout mouse. J. Clin. Invest. 111:1783-1791. doi:10.1172/ JCI200316100.

15. Garg, R., and Yusuf, S. 1995. Overview of randomized trials of angiotensin-converting enzyme inhibitors on mortality and morbidity in patients with heart failure. Collaborative Group on ACE Inhibitor Trials. JAMA. 273:1450-1456.

16. Sharma, D., Buyse, M., Pitt, B., and Rucinska, E.J. 2000. Meta-analysis of observed mortality data from all-controlled, double-blind, multiple-dose studies of losartan in heart failure. Losartan Heart Failure Mortality Meta-analysis Study Group. Am. J. Cardiol. 85:187-192.

17. del Carmen Medina, L., Vazquez-Prado, J., and Garcia-Sainz, J.A. 2000. Cross-talk between receptors with intrinsic tyrosine kinase activity and $\alpha 1 \mathrm{~b}$ adrenoceptors. Biochem. J. 350:413-419.

18. Olivares-Reyes, J.A., et al. 2005. Agonist-induced interactions between angiotensin AT1 and epidermal growth factor receptors. Mol. Pharmacol. 68:356-364. 